ISSN 1991-8631

Original Paper

http://indexmedicus.afro.who.int

\title{
Traitement d'eaux résiduaires urbaines par filtres plantés à flux vertical sous climat Soudano-Sahélien
}

\author{
Martine KONE ${ }^{1 *}$, Inoussa ZONGO ${ }^{1}$, Lucien BONOU $^{3}$, Jean KOULIDIATI ${ }^{3}$, \\ Pierre JOLY ${ }^{4}$, Yvette BOUVET ${ }^{4}$ et Soumaïla SODRE ${ }^{2}$ \\ ${ }^{I}$ Institut de Recherche en Sciences Appliquées et Technologies / CNRST, 03 BP 7047 Ouagadougou 03, \\ Burkina Faso. \\ ${ }^{2}$ Office National de l'Eau et de l'Assainissement (ONEA), 01 BP 170 Ouaga 01, Burkina Faso. \\ ${ }^{3}$ UFR/SEA, Université de Ouagadougou, 03 BP 7021 Ouagadougou 03, Burkina Faso. \\ ${ }^{4}$ Laboratoire d'Ecologie des Hydrosystèmes Naturels et Antropisés, Université Claude Bernard Lyon1, 43, \\ Boulevard du 11 nov. 1918, 69622 Villeurbanne, France. \\ *Auteur correspondant, E-mail : koneba2003@yahoo.fr, Tél : +22650356031/+22670307668
}

\section{RESUME}

Les eaux résiduaires urbaines de la ville de Ouagadougou, ont été traitées par filtres plantés d'Androgopon gayanus et Vetiveria nigritana, deux poacées tropicales, afin d'apprécier leurs performances épuratoires. Les plants ont été repiqués à raison de 9 plants $/ \mathrm{m}^{2}$ et deux tiges par trou dans des pilotes. Six mois après, ils ont été alimentés en eaux usées trois jours successifs par semaine et laissés au repos le reste du temps, avec une charge hydraulique de $60 \mathrm{~L} \mathrm{~m}^{-2} \mathrm{j}^{-1}$. Les rendements sur la Demande Chimique en Oxygène (DCO) de 90 et $94 \%$ ont été observés respectivement pour le filtre planté d'A. gayanus et celui planté de $V$. nigritana, alors qu'en termes de Demande Biochimique en Oxygène de cinq jours $\left(\mathrm{DBO}_{5}\right)$, les deux réacteurs s'équivalent avec des rendements moyens de 97,3 et 97,5\%. Les abattements sur l'ammonium sont respectivement de 96 et $97 \%$ pour les deux filtres. S'agissant du phosphore, le pilote planté d'A. gayanus a donné un abattement moyen sur les orthophosphates de $75 \%$ tandis qu'il est de $67 \%$ avec V. Nigritana. La forte évapotranspiration dans la zone d'étude explique les faibles débits restitués et l'augmentation de la conductivité de $68 \%$ pour le premier et de $28 \%$ pour le second.

(C) 2011 International Formulae Group. All rights reserved.

Mots clés : Filtres plantés, Androgopon Gayanus, DCO, azote.

\section{INTRODUCTION}

Le Burkina Faso est un pays en développement à revenus limités où tout est une priorité. Qu'il s'agisse de la santé de l'éducation ou de l'environnement beaucoup reste à faire. Pour utiliser les objectifs du millénaire pour le développement comme un tremplin, un accent particulier est mis sur la problématique de l'assainissement.
C'est dans l'optique de proposer des solutions innovantes et à moindre coût, adaptées aux conditions climatiques et socioéconomiques de ce pays que nous avons expérimenté les filtres plantés en utilisant deux poacées locales utilitaires et bien connues des populations de l'Afrique subsaharienne en général. Il s'agit d'A. gayanus et $V$. nigritana, des plantes fourragères 
utilisées comme haie anti-érosion et pour la restauration des sols entre autres (OuédraogoKoné et al., 2008 ; Champagnat et al., 2006 ; Bilgo et al., 2007).

$\mathrm{Si}$ en Europe le roseau, une autre poacée donne des résultats satisfaisants en traitement des eaux usées, à telle enseigne que les filtres plantés de roseaux sont devenus en France la technologie rustique la plus demandée par les collectivités (Vymazal et al., 2006; Molle et al., 2004 ; Tunçsiper et al., 2006), il nous a semblé pertinent d'évaluer les performances épuratoires de ces deux plantes locales, sous climat soudano sahélien. Dans la zone d'étude, les températures moyennes mensuelles ont varié au cours de la période d'expérimentation (juillet 2009 à février 2010) entre $17,2{ }^{\circ} \mathrm{C}$ et $39,9{ }^{\circ} \mathrm{C}$, tandis que l'évapotranspiration moyenne mensuelle a oscillé entre $145,5 \mathrm{~mm}$ et $207,8 \mathrm{~mm}$.

L'objectif de ce travail est d'étudier les performances comparées des deux poacées sur l'élimination de la pollution organique (DCO et $\mathrm{DBO}_{5}$ ), l'abattement sur les nutriments $\mathrm{N}$ et $\mathrm{P}$, mais également de constater l'évolution des paramètres physico-chimiques que sont le $\mathrm{pH}$ et la conductivité électrique. Les essais ont portés sur des effluents issus d'un bassin anaérobie de la station de traitement par lagunage à microphytes qui reçoit les eaux résiduaires urbaines (ERU) drainées par un réseau séparatif. Ces eaux ont une typologie domestique à dominance agroalimentaire du fait de la connexion au réseau d'une unité de brasserie.

\section{MATERIEL ET METHODES}

\section{Le dispositif expérimental}

Les pilotes sont constitués de deux fûts cubiques d'une capacité d' $1 \mathrm{~m}^{3}$ chacun (Photo 1). Ils sont inclinés de $10^{\circ}$ vers l'avant afin de faciliter la sortie des eaux et éviter une rétention importante. Le sable servant de matériau de filtration est du sable de rivière de granulométrie moyenne avec : $\mathrm{d}_{10}=0,30 \mathrm{~mm}$, $\mathrm{d}_{60}=0,80 \mathrm{~mm}, \mathrm{CU}=\mathrm{d}_{60} / \mathrm{d}_{10}=2,66$ et $0,6 \%$ de fines. Les massifs ont la même composition pour les deux pilotes : FPA (Filtre planté d'A. gayanus), FPV (Filtre planté de $V$. nigritana). De bas en haut, $15 \mathrm{~cm}$ de couche de drainage constitué de granite de diamètre variant entre 20 et $40 \mathrm{~mm}$, Couche de transition : $20 \mathrm{~cm}$ de granite $(10 / 25 \mathrm{~mm})$ suivi de $10 \mathrm{~cm}$ de granite $(3 / 8 \mathrm{~mm})$, Couche de filtration : $50 \mathrm{~cm} \mathrm{de}$ sable $\mathrm{d}_{10}=0,30 \mathrm{~mm}$.

\section{Evolution des plantes}

Les deux plantes herbacées locales $A$. gayanus et $V$. nigritana (reclassé Chrysopogon nigritana) d'une hauteur de $120 \mathrm{~cm}$ en moyenne ont été coupées à $20 \mathrm{~cm}$ avant d'être repiquées le $1^{\text {er }}$ juillet 2009. Elles ont ensuite été arrosées à l'eau claire pendant 23 semaines avant d'être alimentées avec des eaux usées. 18 semaines après le repiquage, A. gayanus était arrivée au terme de son cycle de croissance; Nous avons alors faucardé la biomasse végétale des deux pilotes, et nous avons continué à les alimenter à l'eau claire pendant encore 5 semaines avant de passer à l'alimentation avec l'effluent prélevé à la sortie du bassin anaérobie de la station de lagunage. $\mathrm{Ce}$ faucardage a permis un développement plus important de la biomasse aérienne avec une pousse plus importante de nouvelles tiges à partir de la touffe. La Figure 1 donne l'évolution de la taille des plants avant et après le faucardage. On note un ralentissement plus marqué de la croissance de $V$. nigritana, avec l'alimentation en eaux usées. Cependant, aucune des deux poacées n'a pu atteindre la taille acquise avant faucardage.

\section{Fonctionnement des pilotes}

A partir de la $24^{\text {ème }}$ semaine après repiquage, les pilotes sont alimentés avec les eaux usées issues du bassin anaérobie de lagunage qui fait office d'un traitement primaire assurant un premier abattement sur les Matières en Suspension (MES). Le système fonctionne comme un deuxième étage de traitement par filtre planté. Ils sont alimentés trois jours successifs et mis au repos 
pendant quatre jours pour simuler un fonctionnement en alternance de deux lits en conditions réelles. La charge hydraulique de $60 \mathrm{~L} \mathrm{~m}^{-2} \mathrm{j}^{-1}$ est répartie en deux bâchées à 8 heures et 14 heures 30 minutes pour simuler les pics de consommation d'eau et de production d'eaux usées dans un contexte où les besoins en eau sont plus importants le matin et l'après midi à la reprise des activités. Nous avons un dimensionnement équivalent à 0,8 à $1,2 \mathrm{~m}^{2} / \mathrm{EH}$, en prenant en compte le fait que les concentrations DCO et les MES sont élevées. Le dimensionnement standard préconisé étant de 2 à $2,5 \mathrm{~m}^{2}$ par Equivalent Habitant (EH) (Agence de l'eau, 2005).

Les analyses sont effectuées sur un échantillon moyen de 24 heures, les trois jours successifs d'alimentation.

\section{Méthodes analytiques}

La DCO est déterminée par oxydation au dichromate de potassium en milieu acide conformément à la norme AFNOR T-90-101. La lecture de la valeur de l'absorbance est faite par spectrophotométrie à la longueur d'onde de $600 \mathrm{~nm}$ ou $420 \mathrm{~nm}$ selon la gamme de DCO choisie. $\mathrm{La} \mathrm{DBO}_{5}$ est déterminée par la méthode manométrique avec des manomètres Oxitop WTW, selon la norme NF 90-103. Les concentrations en nitrates, nitrites, ammoniums et orthophasphates sont déterminées par la méthode colorimétrique, la lecture est faite au spectrophotomètre DR/2010 de HACH LANGE. L'azote Kjeldahl est déterminé après minéralisation des matières organiques en milieu acide et en présence de catalyseur puis entraînement à la vapeur en milieu alcalin de l'azote ammoniacal obtenu et dosage par titrimétrie conformément à la norme française NF 90-110. Le pH et la conductivité sont obtenus avec le multimètre multi 340 i de WTW.

Nous avons utilisé le logiciel STATISTICA version 6 pour analyser les résultats. La performance comparée des filtres plantés FPA et FPV est testée avec un niveau de significativité de 0,05 par utilisation du test de Wilcoxon pour échantillons appariés.

\section{RESULTATS}

Typologie des Eaux Résiduaires Urbaines (ERU) admises à la station de traitement

Les eaux usées reçues à la station de lagunage sont issues d'un réseau séparatif qui draine les eaux usées domestiques du centre ville et les rejets d'un abattoir et d'une brasserie. Le Tableau 1 donne les caractéristiques de l'effluent d'alimentation des pilotes, en sortie du bassin anaérobie.

Les rejets de la brasserie qui utilise de la chaux pour l'adoucissement de l'eau entrant dans la fabrication des boissons, par élimination des hydrogénocarbonates de calcium et de magnésium, ont la particularité d'être riches en carbonates de calcium et hydroxydes de magnésium générés par les réactions de décarbonatation. En outre, ils présentent des concentrations très élevées en MES, DCO et $\mathrm{DBO}_{5}$ avec des valeurs moyennes respectives $645 \mathrm{mg} / \mathrm{L}, 3078 \mathrm{mg} \mathrm{O}_{2} / \mathrm{L}$ et $1918 \mathrm{mg} \mathrm{O}_{2} / \mathrm{L}$ sur l'année 2009.

Ces eaux généralement basiques doivent être neutralisées avant admission dans le réseau collectif. Le séjour en bassin anaérobies qui met en œuvre des processus de digestion anaérobie permet une baisse notable du $\mathrm{pH}$ avec des valeurs variant entre 6,8 et 8,9 à la sortie de ce bassin.

En dépit du séjour en bassin anaérobie, les ERU initialement très chargées présentent des valeurs assez élevées en DCO, $\mathrm{DBO}_{5}$ et $\mathrm{NK}$ avec des moyennes respectives de $1298 \mathrm{mg}$ $\mathrm{O}_{2} / \mathrm{L}, 886 \mathrm{mg} \mathrm{O}_{2} / \mathrm{L}$ et $61,63 \mathrm{mg} / \mathrm{L}$, compte tenu du fait que plus de $50 \%$ des charges hydrauliques entrant à la station sont issues de la brasserie. Néanmoins, le ratio des valeurs

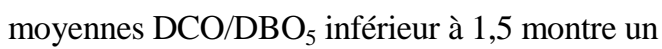
caractère très biodégradable de l'effluent d'alimentation des pilotes. En général, les valeurs des différents paramètres mesurés sur l'effluent issu du bassin anaérobie varient peu dans le temps (Tableau 1). En effet, il y'a un lissage des teneurs lié au temps de séjour en 
anaérobiose, qui a tendance à être long compte tenu des débits faibles en entrée de station, puisque celle-ci fonctionne en sous charge.

\section{Performances épuratoires des massifs La pollution carbonée}

La Demande Chimique en Oxygène : La Figure 4 présente l'évolution de la DCO dans le temps au niveau des deux pilotes. Les rendements épuratoires moyens sont respectivement de 90 et $94 \%$ pour FPA et FPV, avec des teneurs en DCO variant entre $51 \mathrm{mg}$ $\mathrm{O}_{2} / \mathrm{L}$ et $273 \mathrm{mg} \mathrm{O}_{2} / \mathrm{L}$ pour le premier et une DCO variant entre $43 \mathrm{mg} \mathrm{O} / 2$ et $163 \mathrm{mg} \mathrm{O}_{2} / \mathrm{L}$ pour le second. L'analyse statistique des variables donne une différence significative entre les performances de FPA et FPV avec $\mathrm{p}<$ 0,05. Morani et Giardini (2009), ont relevé des rendements supérieurs à $86 \%$ en $\mathrm{DCO}$ et $\mathrm{DBO}_{5}$ avec des filtres plantés de Typha latifolia et phragmites australis. Ouattara et al. (2008), ont obtenu des rendements de $91 \%$ à partir de travaux similaires sur Panicum maximum sous climat tropical. Il est important de noter que les valeurs des différents paramètres sont impactées par les débits restitués par les pilotes, ceux-ci étant plus faibles pour FPA (Figure 3)

La Demande Biochimique en Oxygène : La variation de la $\mathrm{DBO}_{5}$ des filtrats issus des deux pilotes est donnée par la Figure 5. On note une différence significative $(p<0,05)$ entre les comportements des deux filtres avec des rendements épuratoires sur les teneurs en $\mathrm{DBO}_{5}$, variant entre 95 et $98 \%$ pour le filtre planté d'A. gayanus, tandis qu'au niveau du filtre planté de $V$. nigritana ils sont compris entre 96 et $99 \%$ avec des teneurs moyennes en $\mathrm{DBO}_{5}$ respectives de 26 et $22 \mathrm{mg} \mathrm{O}_{2} / \mathrm{L}$, alors que l'effluent brut d'alimentation présente une concentration moyenne en $\mathrm{DBO}_{5}$ de $886 \mathrm{mg} \mathrm{O}_{2}$ /L. A l'inverse, lorsque l'on considère les charges en n'occultant pas les besoins en eau plus importants d'A. gayanus par rapport à $V$. nigritana, on constate que les charges rejetées par FPV sont plus importantes que celles rejetées par FPA avec des abattements sur les charges journalières moyennes respectifs de 98\% et $99 \%$.

\section{La pollution azotée}

La nitrification: S'agissant de la nitrification, l'analyse statistique des variables révèle une différence non significative $(p>0,05)$, avec une concentration moyenne légèrement supérieure au niveau du réacteur FPV, $36 \mathrm{mg}$ $\mathrm{N}-\mathrm{NO}_{3} / \mathrm{L}$, comparativement à celle de FPA qui est de $35 \mathrm{mg} \mathrm{N}-\mathrm{NO}_{3} / \mathrm{L}$ (Figure 6). Lorsque nous considérons les charges journalières moyennes en nitrates en sortie de pilotes, qui sont de1388 $\mathrm{mg} \mathrm{N}-\mathrm{NO}_{3} / \mathrm{j}$ pour le premier et $849 \mathrm{mg} \mathrm{N}-\mathrm{NO}_{3}$ /j pour le second la différence de performance est plus nette. Une bonne nitrification nécessite des conditions favorables d'oxygénation. L'alimentation par bâchées d'une part et le temps de repos d'autre part favorisent la recharge en oxygène du massif (Winter and Goetz, 2003; Obarska-Pempkowiak and Gajewska, 2005), même si par ailleurs il y'a une compétition entre la nitrification et l'élimination de la pollution carbonée dans l'utilisation de l'oxygène par les microorganismes (Molle et al., 2004). La présence des plantes a aussi un impact sur le processus de dégradation en ce sens que les racines sont le lieu privilégié de développement des bactéries (Münch et al., 2004). Tunçsiper et al. (2006) rapportent des taux moyens annuels d'abattement sur l'ammonium, les nitrates et l'azote organique respectifs de 76,59 et $75 \%$ avec des filtres plantés à flux vertical.

L'ammonium: Du point de vue statistique, on note tout comme avec les nitrates, une différence non significative $(p>0,05)$ s'agissant des concentrations en ammonium relevées en sortie des pilotes. En effet, les teneurs moyennes relevées sont faibles et très peu différentes 1,28 $\mathrm{mg} \mathrm{N}-\mathrm{NH}_{4} / \mathrm{L}$ et $1,07 \mathrm{mg} \mathrm{N}$ $\mathrm{NH}_{4} / \mathrm{L}$ respectivement avec FPA et FPV contre des teneurs à l'entrée de $31 \mathrm{mg} \mathrm{N}-\mathrm{NH}_{4} / \mathrm{L}$. Cela dénote ainsi un bon abattement sur ce paramètre ( $96 \%$ et $97 \%$ de rendements) qui se traduit par le niveau de nitrification observé plus haut, même si par ailleurs l'ammonium a des capacités de s'adsorber sur le massif en partie. 
La dégradation de l'azote ammoniacal est tributaire des conditions d'aération du massif mais également des charges hydrauliques apportées, de la granulométrie du matériau filtrant et de sa hauteur (Kantawanichkul et al., 2009).

Les nitrites : L'effluent d'alimentation ne présentait pas de nitrites. Cependant, nous avons relevé de faibles teneurs en nitrites en sortie de pilotes avec une valeur moyenne de $1,00 \mathrm{mg} \mathrm{N}$ $\mathrm{NO}_{2} / \mathrm{L}$ pour FPA et $0,16 \mathrm{mg} \mathrm{N}-\mathrm{NO}_{2} / \mathrm{L}$ pour FPV.

L'azote Kjeldahl (NK) : Les eaux usées à l'entrée des pilotes avaient une teneur moyenne en $\mathrm{NK}$ de $61,63 \mathrm{mg}$ de $\mathrm{N} / \mathrm{L}$ tandis qu'en sortie de pilotes nous avons enregistré comme valeurs moyennes 16,75 et $14,02 \mathrm{mg}$ N/L (Tableau 2) au niveau de FPA et FPV correspondant à des rendements respectifs de 73 et $77 \%$ sur l'abattement de NK avec une significativité $\mathrm{p}>0,05$. L'azote Kjeldahl étant constitué des formes réduites de l'azote (organique et ammoniacal) donc celles susceptibles d'être oxydées.

\section{Les orthophosphates}

La Figure 7 indique un abattement sur les concentrations plus important pour FPA par rapport à $\mathrm{FPV}$, avec des rendements moyens de $75 \%$ et $67 \%$ respectivement avec une différence significative $(\mathrm{p}<0,05)$ à l'issue de l'analyse statistique des variables.

Les résultats des travaux de Ouattara et al. (2008) sont en accord avec les nôtres. En effet, ayant travaillé sur panicum maximum sous climat tropical, ils ont obtenu un rendement moyen sur les orthophosphates de $75 \%$, contre $49 \%$ pour un témoin non planté. Ils attribuent cette performance à l'assimilation bactérienne et /ou végétale, mais également à l'adsorption dans le massif filtrant tout comme Molle (2003). Ouattara et al. (2008) ont relevé un rendement épuratoire sur $\mathrm{PO}_{4}{ }^{3-}$ avec Panicum maximum supérieur à ceux obtenus par Coulibaly et al. (2008) qui ont travaillé sur Amaranthus hybridus et Corchorus oliterius.

Les charges moyennes évacuées durant les trois jours successifs $14 \mathrm{mg} / \mathrm{j}, 76 \mathrm{mg} / \mathrm{j}$ et 87 mg/j pour FPA contre $55 \mathrm{mg} / \mathrm{j}, 134 \mathrm{mg} / \mathrm{j}$ et 133 $\mathrm{mg} / \mathrm{j}$ pour FPV montrent des valeurs plus faibles les premiers jours d'alimentation, avec un impact plus marqué pour le pilote planté d'A. gayanus.

\section{Les paramètres physico-chimiques}

Le $\mathrm{pH}$ : Le $\mathrm{pH}$ de l'effluent d'alimentation a varié entre 6,8 et 7,5 durant la période d'expérimentation. Au niveau des deux pilotes nous avons constaté une augmentation du $\mathrm{pH}$ qui cependant est restée dans une certaine fourchette de valeurs proches de 8. En effet, le filtrat issu du pilote planté d'A. gayanus a donné des $\mathrm{pH}$ compris entre 7,9 et 9,1 avec une moyenne de 8,5, tandis que celui du second réacteur a varié entre 7,6 et 8,7 avec une moyenne de 8,2 . On note une alcalinisation du milieu qui est légèrement plus importante le premier jour d'alimentation par rapport aux deux jours suivants (Figure 8), et ce aussi bien avec FPA que FPV même si le phénomène est plus marqué avec le pilote planté d'A. gayanus qui donne des $\mathrm{pH}$ légèrement plus élevés en comparaison des valeurs relevés au niveau du réacteur FPV. Le test de Wilcoxon pour échantillons appariés indique une différence significative $(\mathrm{p}<0,05)$.

Conductivité électrique: La Figure 9 présente une augmentation de la conductivité au niveau des deux réacteurs biologiques passant d'une moyenne de $1936 \mu \mathrm{S} / \mathrm{cm}$ de l'effluent d'alimentation à des moyennes respectives de $3240 \mu \mathrm{S} / \mathrm{cm}$ et $2476 \mu \mathrm{S} / \mathrm{cm}$ pour FPA et FPV. Une fois de plus le pilote planté d'A. gayanus présente les valeurs les plus élevées. On note une baisse de la conductivité les trois jours successifs. En moyenne, les conductivités ont augmenté respectivement de $68 \%$ et $28 \%$ pour FPA et FPV. En dépit de l'adsorption de certains ions sur le support granulaire et des prélèvements des plantes comme nutriments, les valeurs de conductivité sont restées supérieures à celles de l'ERU et ce pour les deux filtres plantés. L'analyse statistique indique une différence significative avec $\mathrm{p}<0,05$. 


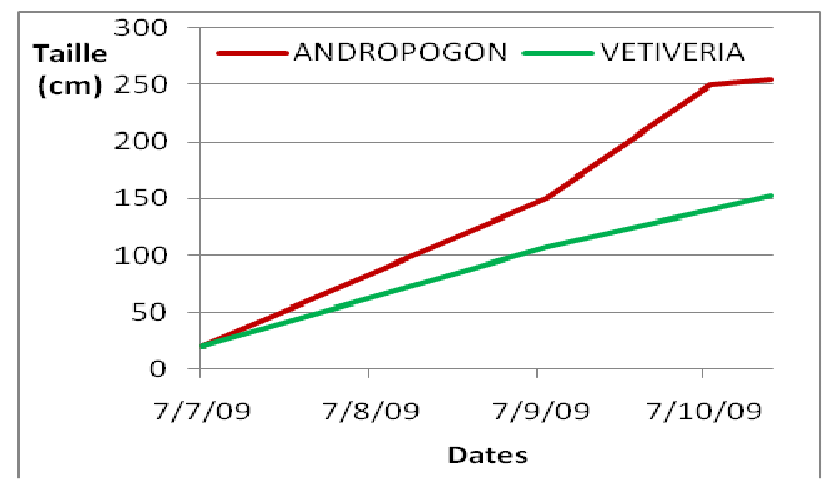

Figure 1a : Evolution des plants après repiquage sans alimentation à l'eau usée Evolution temporelle de la taille des plantes avant et après le faucardage.

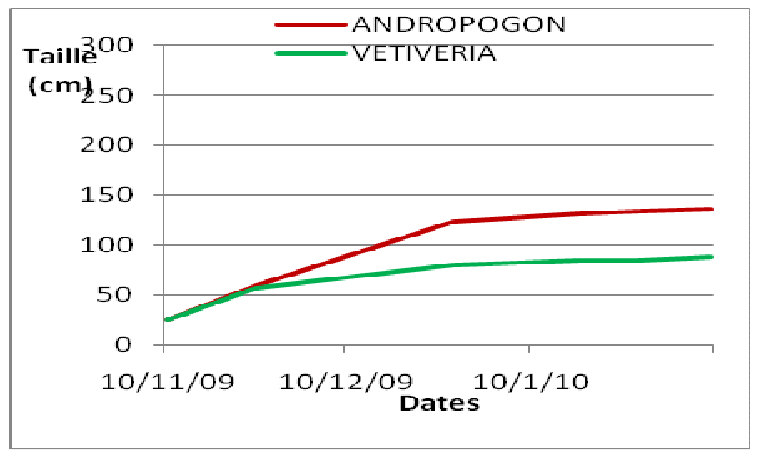

Figure 1b : Evolution des plants après le faucardage suivi de l'alimentation à l'eau usée Aucune des deux graminées n'a atteint sa taille maximale d'avant faucardage.

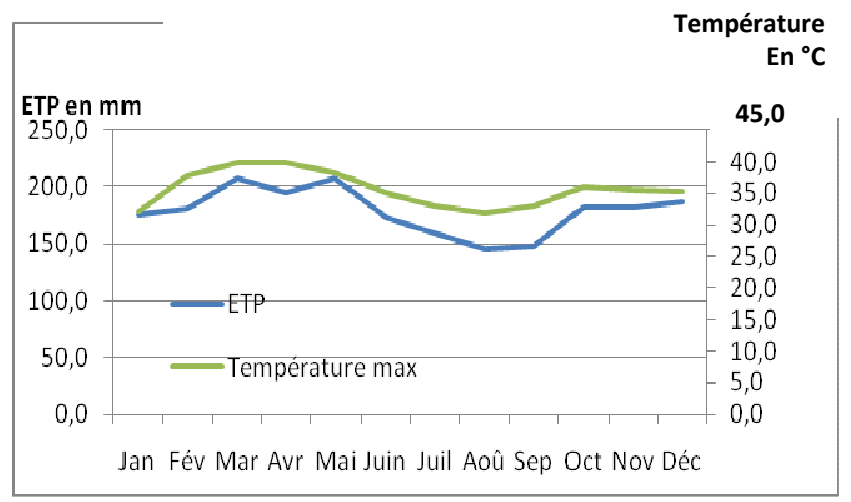

Figure 2 : Courbes d'évolution de la température et de l'évapotranspiration au Burkina Faso au cours de l'année 2009.

L'évapotranspiration augmente avec la température, et janvier à avril constitue la période de fortes températures et d'évapotranspiration. (Source météo Burkina Faso). 


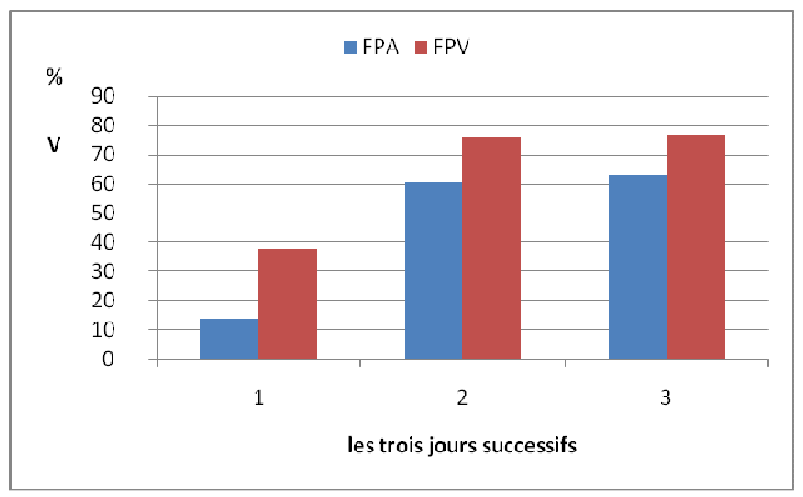

Figure 3 : Evolution du pourcentage des débits moyens restitués les trois jours successifs d'alimentation.

La quantité de filtrat recueilli le premier jour est la plus faible des trois jours successifs pour les deux pilotes. Andropogon gayanus (FPA) présente une évapotranspiration plus importante avec seulement 14\% de la charge restituée ce jour contre $38 \%$ pour Vetiveria nicritana (FPV).

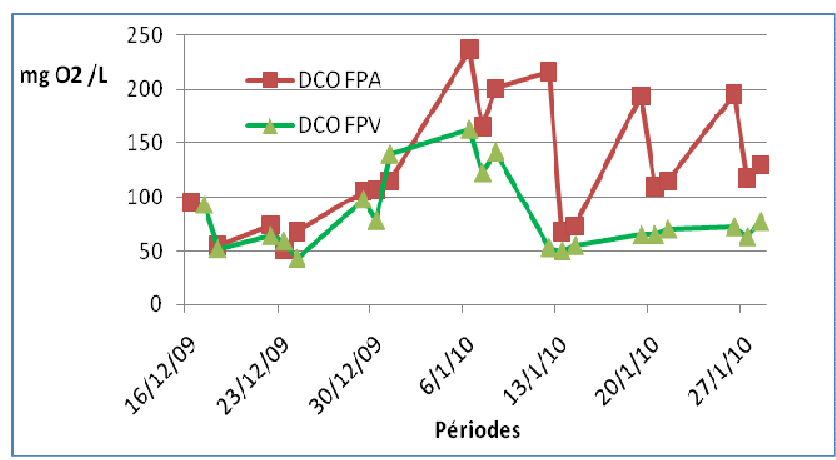

Figure 4 : Variation temporelle de la DCO au niveau du filtre planté d'Andropogon gayanus (FPA) et du filtre planté de Vetiveria nigritana (FPV).

La DCO tend à augmenter avec le temps avec la baisse de plus en plus importante du débit restitué particulièrement à la reprise de l'alimentation. Cela est plus marqué pour Andropogon gayanus que pour Vetiveria nigritana.

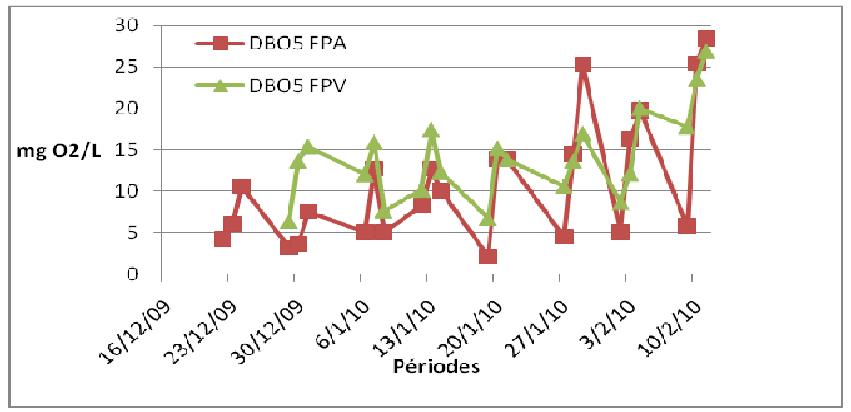

Figure 5 : Variation temporelle de la $\mathrm{DBO}_{5}$ au niveau de FPA et FPV. Les deux graminées assurent des abattements comparables sur la $\mathrm{DBO}_{5}$. 


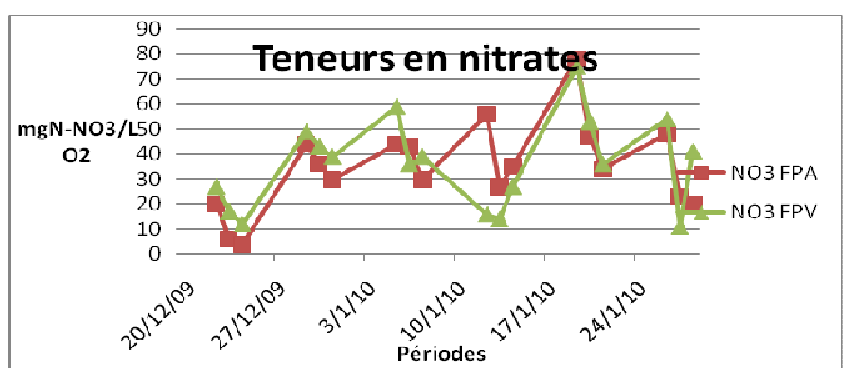

Figure 6 : Variation temporelle des teneurs en nitrates relevées au niveau de FPA et FPV. Les teneurs en nitrates suivent la même évolution dans le temps au niveau des deux pilotes.

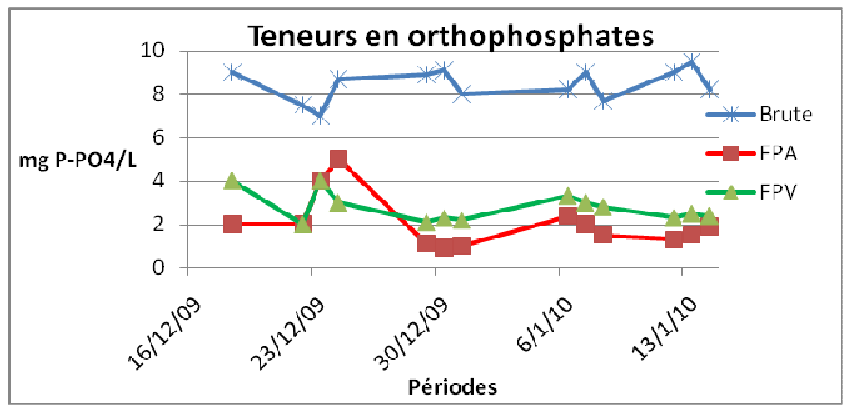

Figure 7 : Variation temporelle des teneurs en orthophosphates relevées au niveau de FPA et FPV. L'abattement sur les orthophosphates est plus important avec Andropogon gayanus, bien qu'il restitue les débits les plus faibles.

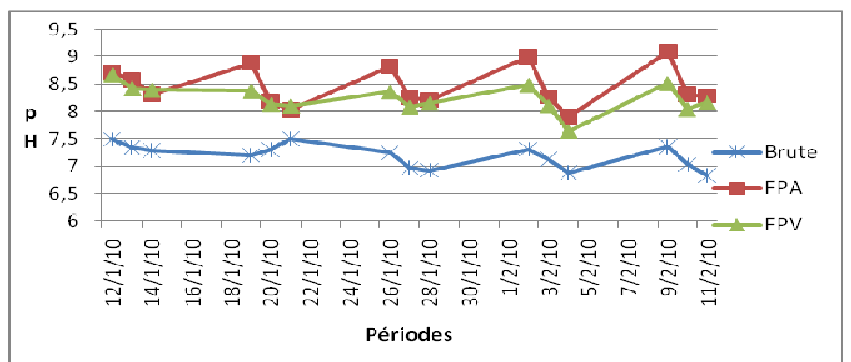

Figure 8 : Evolution temporelle des $\mathrm{pH}$ au niveau des deux pilotes en rapport avec ceux de l'effluent d'alimentation.

Le milieu devient alcalin au sein des deux massifs. Le phénomène est plus marqué à la reprise de l'alimentation pour les deux pilotes. L'effluent issu du pilote planté d' Andropogon gayanus est plus alcalin.

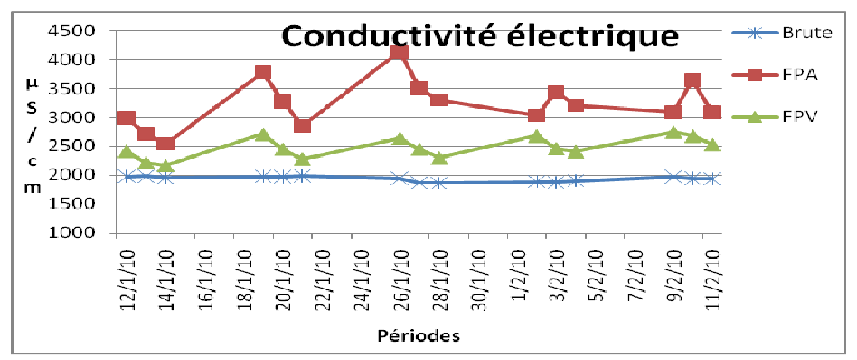

Figure 9 : Evolution temporelle de la conductivité électrique au niveau des deux pilotes en rapport avec les valeurs de l'effluent d'alimentation.

La conductivité augmente par rapport à celle de l'effluent d'alimentation pour les deux pilotes. La hausse de conductivité est plus importante à la reprise de l'alimentation et le filtre planté d'Andropogon gayanus présente les plus fortes valeurs de conductivité. 


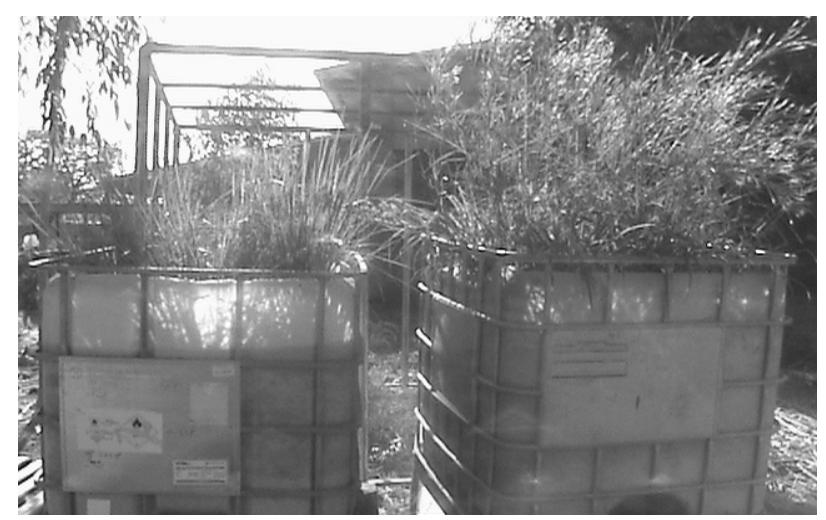

Photo 1 : Les pilotes constitués de deux fûts cubiques d'une capacité d'1 $\mathrm{m}^{3}$ chacun.

Tableau 1 : Valeurs caractéristiques des paramètres mesurés pour l'effluent d'alimentation.

\begin{tabular}{lccccc}
\hline Paramètres & $\begin{array}{c}\text { Nombre } \\
\text { d'échantillons }\end{array}$ & $\begin{array}{c}\text { Valeur } \\
\text { minimale }\end{array}$ & $\begin{array}{c}\text { Valeur } \\
\text { maximale }\end{array}$ & Moyenne & Ecart type \\
\hline $\mathrm{DCO}(\mathrm{mg} \mathrm{O} / \mathrm{L})$ & 47 & 1073 & 1580 & 1298 & 180 \\
$\mathrm{DBO} 5(\mathrm{mg} \mathrm{O} / \mathrm{L})$ & 47 & 980 & 756 & 886 & 67,16 \\
$\mathrm{NO} 3((\mathrm{mg} / \mathrm{L})$ & 37 & 0,30 & 4,60 & 1,82 & 0,71 \\
$\mathrm{NO}_{2}{ }^{-}(\mathrm{mg} / \mathrm{L})$ & 37 & 00 & 00 & 00 & \\
$\mathrm{NH}_{4}{ }^{-}(\mathrm{mg} / \mathrm{L})$ & 37 & 19,00 & 39,00 & 31,00 & 5,10 \\
$\mathrm{NK}^{(\mathrm{mg} / \mathrm{L})}$ & 37 & 47,44 & 71,00 & 61,63 & 9,14 \\
$\mathrm{PO}_{4}{ }^{3-}(\mathrm{mg} / \mathrm{L})$ & 26 & 6,40 & 9,50 & 8,20 & 0,784 \\
$\mathrm{pH}^{-}$ & 37 & 6,82 & 8,88 & 7,60 & 0,603 \\
$\begin{array}{l}\mathrm{Conductivité} \\
(\mu \mathrm{S} / \mathrm{cm})\end{array}$ & 37 & 1023 & 1985 & 1580 & 363 \\
\hline
\end{tabular}

Tableau 2 : Valeurs moyennes et écart-types des paramètres caractéristiques du filtrat à la sortie des pilotes.

\begin{tabular}{lcccccc}
\hline Paramètres & Pilote & $\begin{array}{c}\text { Nombre } \\
\text { d'échantillons }\end{array}$ & $\begin{array}{c}\text { Valeur } \\
\text { minimale }\end{array}$ & $\begin{array}{c}\text { Valeur } \\
\text { maximale }\end{array}$ & Moyenne & Ecartype \\
\hline $\mathrm{DCO}\left(\mathrm{mg} \mathrm{O}_{2} / \mathrm{L}\right)$ & FPA & 24 & 51 & 237 & 122 & 61 \\
& FPV & 24 & 30 & 163 & 77 & 37 \\
\hline $\mathrm{DBO}_{5}\left(\mathrm{mg} \mathrm{O}_{2} / \mathrm{L}\right)$ & FPA & 24 & 8 & 45 & 26 & 10 \\
& FPV & 24 & 9 & 40 & 22 & 8 \\
\hline $\mathrm{NO}_{3}{ }^{-}(\mathrm{mg} / \mathrm{L})$ & FPA & 18 & 14 & 58 & 35 & 13,81 \\
& FPV & 18 & 17 & 55 & 36 & 14,47 \\
\hline
\end{tabular}




\begin{tabular}{lllcccc}
\hline $\mathrm{NH}_{4}{ }^{-}(\mathrm{mg} / \mathrm{L})$ & FPA & 18 & 1 & 2 & 1,28 & 0,47 \\
& FPV & 18 & 0 & 2 & 1,07 & 0,52 \\
\hline $\mathrm{NO}_{2}{ }^{-}(\mathrm{mg} / \mathrm{L})$ & FPA & 12 & 1,40 & 0,7 & 1,00 & 0,30 \\
& FPV & 12 & 1,00 & 00 & 0,16 & 0,31 \\
\hline $\mathrm{NK}(\mathrm{mg} / \mathrm{L})$ & FPA & 12 & 9,50 & 21,90 & 16,75 & 4,33 \\
& FPV & 12 & 11,60 & 15,90 & 14,02 & 1,40 \\
\hline $\mathrm{PO}_{4}{ }^{3-}(\mathrm{mg} / \mathrm{L})$ & FPA & 18 & 0,90 & 5,00 & 2,04 & 1,20 \\
& FPV & 18 & 2,00 & 4,00 & 2,76 & 0,67 \\
\hline $\mathrm{pH}$ & FPA & 18 & 7,90 & 9,09 & 8,45 & 0,37 \\
& FPV & 18 & 7,64 & 8,67 & 8,24 & 0,25 \\
\hline Conductivité & FPA & 18 & 2540 & 4140 & 3240 & 418 \\
$(\mu \mathrm{S} / \mathrm{cm})$ & FPV & 18 & 2170 & 2750 & 2477 & 185 \\
\hline
\end{tabular}

\section{DISCUSSION}

\section{L'élimination de la pollution La pollution carbonée}

La DCO: Si les deux réacteurs ont évolué de façon similaire les trois premières semaines, dès la quatrième semaine FPA a montré une plus grande tendance à l'augmentation de la DCO, particulièrement à la reprise de l'alimentation. Parallèlement à cette évolution de la DCO, les besoins en eau d'A. gayanus sont plus élevés comparativement à ceux de $V$. nigritana surtout le premier jour d'alimentation des pilotes avec $14 \%$ de débit restitué par FPA contre $38 \%$ pour FPV soient respectivement 8 et 23 litres (Figure 3).

Cet état de fait peut être attribué en partie à la réhumectation du massif asséché avec la phase de repos. Les forces osmotiques et matricielles y sont importantes particulièrement celles liées à l'imbibition, d'autant plus que le massif est riche en sels minéraux adsorbés à partir des eaux usées. L'évapotranspiration, très importante à cette période de l'année (Janvier février) au Burkina Faso (Figure 2) et l'élévation des températures augmentant la transpiration au niveau des plantes, pourraient expliquer les pertes en eau très importantes à la réalimentation des massifs et par voie de conséquence la forte concentration en DCO du premier jour d'alimentation. Les résultats des travaux de Morani et Giardini (2009), portant sur les performances épuratoires de Typha latifolia et Phragmites australis, confirment l'impact majeur de l'évapotranspiration sur la qualité de l'effluent à l'issue du traitement. En effet, les plantes ayant connu un stress hydrique de 4 jours présentent un potentiel hydrique très faible qui se traduit par un flétrissement réversible des feuilles et un appel d'eau, d'autant plus important que les plants sont en croissance. Le stress hydrique subit par les plantes est variable selon qu'il s'agit d'A. gayanus ou de $V$. nigritana, le second résistant mieux aux conditions climatiques difficiles. C'est ainsi qu'à la date du 25 février, après un temps de séjour exceptionnel de 5 jours, $V$. nigritana avait toujours ses feuilles vertes et fraiches, alors qu'A. gayanus avait atteint un stade de flétrissement extrême de non retour du fait de l'élévation de plus en plus importante de la température qui atteignait $40^{\circ} \mathrm{C}$ à l'ombre à cette période, nous obligeant 15 semaines après le précédent faucardage à procéder à un nouveau faucardage. Notons cependant que dans des conditions similaires Kantawanichkul et al. (2009), ont eux aussi obtenu avec des filtres plantés de Typha angustifolia $L$. et involucratus Cyperus Rottb des performances peu différentes de celles d'un témoin non planté.

$\mathrm{La} \quad \mathrm{DBO}_{5}:$ Les deux réacteurs présentent de très bonnes performances liées à une bonne colonisation des massifs par les microorganismes épurateurs, notamment autour du système racinaire deux fois plus développé chez $A$ gayanus $(60 \mathrm{~cm})$ par rapport à $V$. nigritana $(31 \mathrm{~cm})$, mais aussi grâce à une bonne oxygénation du massif avec les phases d'alimentation et de repos (Liénard et al., 2005). En effet, le système non saturé favorise 
l'aération, mais les phases de repos permettent une recharge plus importante du massif en oxygène, utile au métabolisme des bactéries lors de la biodégradation de la matière organique. Des travaux similaires font état de rendements épuratoires sur la pollution organique de $90 \%$ et plus (Obarska-Pempkowiak et Gajewska, 2005 ; Ouattara et al., 2008). En outre, les échanges sol-plante-bactéries sont favorisés par l'effet rhizosphère qui existe autour des racines qui constituent un support préférentiel pour les bactéries, parce que la zone est mieux oxygénée d'une part et d'autre part du fait que les eaux percolent par des chemins créés le long des racines (Liénard et al., 2005). La matière minérale rendue disponible par l'action des bactéries est utilisée par les plantes pour leurs besoins nutritionnels.

A l'image de la DCO on observe une augmentation des concentrations en $\mathrm{DBO}_{5}$ avec le temps, due sans doute au développement du système racinaire qui facilite la percolation des eaux mais avec l'effet conjugué d'une diminution progressive des prélèvements de nutriments par les plantes qui à cette phase semble avoir arrêté de croître pour ce qui est de $V$. nigritana ou atteignant la fin de croissance pour A. gayanus qui portait à nouveau des épis. Cependant, l'évapotranspiration demeure le facteur le plus important de la concentration des effluents en sortie de pilotes (Kantawanichkul et al., 2009). La Figure 1, permet de constater qu'avec l'alimentation en eaux usées, $A$. gayanus crô̂t plus vite que $V$. nigritana qui, très tôt connaît une stagnation de sa croissance. Cependant, aucun des deux macrophytes n'a atteint la taille acquise avant le faucardage suivi de l'alimentation avec les eaux usées. L'avantage du faucardage avant le stockage des nutriments dans les racines est de donner à la plante une plus grande capacité d'élimination de la pollution avec la croissance de la biomasse végétale (Vymazal, 2004). La forte salinité des eaux et le caractère alcalin du milieu ont eu pour conséquence un ralentissement de la croissance des plantes, même si les effets semblent impacter différemment les deux poacées. En effet, le faucardage a favorisé une augmentation des besoins en eau et en nutriments, prélevés à partir des eaux usées chez A. gayanus qui a crû très vite et a donné une biomasse végétale importante, atteignant ainsi la fin de son cycle de croissance (Morani et Giardini, 2009). Par contre, V. nigritana dont les feuilles présentaient un stress hydrique moins important même après quatre jours de temps de repos, a stagné très tôt et n'avait pas encore atteint la floraison quand A. gayanus a atteint la fin de son cycle de croissance. C'est ainsi que le pilote planté de $V$. nigritana restituait un volume d'eau plus important le premier jour d'alimentation, $38 \%$ contre $14 \%$ avec A. gayanus pour des charges hydrauliques d'alimentation de $60 \mathrm{~L} / \mathrm{m}^{-2} \mathrm{j}^{-1}$ (Figure 3).

En somme, contrairement à $V$. nigritana, A. gayanus tolère mieux l'irrigation avec ces eaux usées caractérisées par une grande salinité et une alcalinité liées à sa richesse en hydroxydes et carbonates de calcium et magnésium, bien que de typologie domestique.

\section{La pollution azotée}

Les teneurs élevées en nitrates ( 35 et 36 $\mathrm{mg} / \mathrm{L}$ respectivement avec FPA et FPV), et faibles en $\mathrm{NH}_{4}^{-}(1,28$ et $1,07 \mathrm{mg} / \mathrm{L})$ associées à des rendements sur NK de 73 et $77 \%$ en sorties de pilotes montrent qu'il y'a une bonne nitrification au sein des deux massifs, ce qui est le signe d'une bonne aération des réacteurs biologiques. Cette performance est attribuable $\mathrm{au}$ développement du système racinaire des plantes mais surtout à l'alternance des phases d'alimentation et de repos qui favorisent la recharge des massifs en oxygène (Molle et al., 2004; Obarska-Pempkowiak et Gajewska, 2005). Ce régime permet également une autorégulation de la biomasse épuratrice par l'installation temporaire d'une disette qui augmente la respiration endogène, étant entendu que la présence des racines et de la biomasse aérienne créent les conditions idéales pour l'accroissement de la population bactérienne (Liénard et al., 2005 ; Agence de l'eau, 2005).

Globalement FPV présente des charges journalières en nitrates plus élevées confirmant ainsi un prélèvement plus important de matière minérale par A. gayanus (Morani et Giardini, 2009), ce d'autant plus que les abattements sur l'ammonium sont pratiquement les mêmes, 
quand bien même, une partie de l'ammonium aurait pu être adsorbée sur le massif. En tout état de cause, la différence de comportement des deux réacteurs ne peut qu'être attribuable aux caractéristiques intrinsèques de chaque plante, puisque les massifs ont la même composition et fonctionnent sous les mêmes conditions. Ces résultats sont en accord avec ceux obtenus par Morani et Giardini (2009) qui ont noté un prélèvement de $\mathrm{N}, \mathrm{P}$ et $\mathrm{K}$ de plus de $65 \%$ par rapport aux teneurs en entrée. Kantawanichkul et al. (2009), ont rapporté une élimination plus importante des nitrates et orthophosphates avec des lits plantés comparativement à un témoin non planté. Kengne et al. (2008) font état d'une élimination différenciée sur l'azote et le phosphore en utilisant Echinochloa pyramidalis et Cyperus papyrus.

Globalement, sur l'élimination de l'azote en termes de teneurs rejetées indépendamment des débits restitués, les deux réacteurs s'équivalent, ce d'autant plus que l'analyse statistique a donné une différence non significative sur les nitrates, l'ammonium et l'azote Kjeldahl. Pour une réutilisation des eaux en agriculture, V.nigritana constitue un meilleur choix en termes de charges hydrauliques restituées mais également de la disponibilité plus importante de nutriments. Cependant, le faucardage a un impact important sur le comportement des poacées qui dans les conditions naturelles, grâce aux stocks de nutriments constitués résistent à des conditions difficiles de sècheresse et de rareté de nutriments.

\section{Les orthophosphates}

La différence de comportement constatée entre FPA et FPV pourrait être liée au prélèvement effectué par les plantes, puisque $\mathrm{PO}_{4}{ }^{3-}$ sert de nutriment aux végétaux. En effet, même si l'abattement est dû en partie à l'adsorption des ions orthophosphates sur le support granulaire, la différence constatée entre les charges moyennes journalières rejetées est due à la plus grande absorption par A. gayanus de ces ions qui sont prélevés au niveau des poils absorbants des racines et acheminés par la sève brute vers les différents organes de la plante.
Cette hypothèse est confortée par la croissance plus rapide d'A. gayanus et ses besoins en eau relativement plus importants (Figure 3).

A. gayanus absorbe donc plus de nutriments $\mathrm{N}$ et $\mathrm{P}$, absorption favorisée par la disponibilité de l'eau. La réserve de nutriments est constituée dans le système racinaire les premières années de son installation, tandis que la plante âgée présente des teneurs plus importantes en nutriments dans la biomasse aérienne. De façon générale, le stock d'azote et de phosphore augmente progressivement jusqu'à la floraison et ce parallèlement à la croissance de la biomasse. On observe ensuite une baisse progressive des réserves jusqu'à la fin du cycle, puisqu'à partir de la floraison les prélèvements de $\mathrm{N}$ et $\mathrm{P}$ sont quasi nuls.

Ouattara et al. (2008) ayant travaillé sur Panicum maximun sous climat tropical ont relevé également une baisse des besoins de la plante en $\mathrm{PO}_{4}{ }^{3-}$ lorsque celle-ci atteint sa croissance maximale, se traduisant par une diminution du rendement d'élimination au niveau du lit planté. A l'inverse, les travaux menés sur Phragmites australis, utilisé couramment dans les filtres plantés en France, montrent des prélèvements très faibles de nutriments tant et si bien que l'intérêt majeur du faucardage revient à réduire l'accumulation de dépôts de MES à la surface des massifs (Liénard et al., 2005).

\section{Evolution des paramètres physico-chimiques}

Le $\mathrm{pH}$ : Comme l'indique la Figure 8, il y'a une alcalisation du milieu qui pourrait relever de différents facteurs dont le caractère «dure » de l'effluent d'alimentation qui faut-il le rappeler est constitué en grande partie des rejets d'une brasserie qui procède à une décarbonatation à la chaux des eaux utilisées dans la fabrication de la bière. Un processus qui génère des précipités de carbonates de calcium et d'hydroxyde de magnésium, évacués avec les eaux usées. Ces composés adsorbés en partie sur le support granulaire sont source d'alcalinisation du milieu par les interactions réactionnelles à l'interface support-plantemicro-organismes. La présence de $\mathrm{CO}_{2}$ issu de la biodégradation de la matière peut remettre en solution de l'hydrogénocarbonate de calcium et 
de magnésium qui sont des formes solubles. Un autre facteur pouvant entrainer une augmentation des $\mathrm{pH}$ provient du métabolisme des plantes qui comme cela a été signalé plus haut absorbent des nutriments à partir des eaux usées reçues. Globalement un équilibre électrostatique doit demeurer au sein des cellules végétales, c'est-à-dire que la somme des charges négatives portées par les anions doit être égale à la somme des charges positives portées par les cations. Par conséquent, un transport d'anions entraine un co-transport de cations à l'intérieur de la plante ou un contre transport d'un autre type d'anion. C'est ainsi que l'absorption d'ions nitrates à travers les racines, se fait à contre courant d'un transport d'ions hydroxydes de la plante vers l'extérieur ou un co-transport d'ions hydroniums à l'intérieur de la cellule végétale (Heller et al., 1998). Dans tous les cas, il y'a une augmentation du pH du milieu.

Néanmoins, il s'avère que c'est la présence des ions carbonates qui est le facteur le plus important pouvant expliquer l'alcalinisation systématique du milieu pendant toute la durée du fonctionnement des pilotes, une hypothèse confortée par le fait que des massifs de sable non plantés, alimentés au même régime avec le même effluent ont donné des filtrats de $\mathrm{pH}$ plus alcalins avec une moyenne de 8,94.

La conductivité : L'augmentation de la conductivité pourrait s'expliquer par la minéralisation de la matière organique qui se traduit du reste par la faible $\mathrm{DBO}_{5}$ notée plus haut, inhérente à l'activité de la flore microbienne au sein du massif et particulièrement dans la zone rhizosphère. Cependant, la typologie des eaux d'alimentation riches au départ en sels minéraux, ainsi que la forte évapotranspiration (Figure 2) sont des facteurs importants dans ce processus. La décroissance des valeurs de conductivité les trois jours successifs confirme l'hypothèse de l'impact de l'évapotranspiration (Figure 9).

En outre, on remarque que si le premier jour d'alimentation des pilotes le débit restitué par FPA est faible comparativement à celui de FPV (14\% contre 38\%), les débits restitués les deux jours suivants sont respectivement de $61 \%$ et $63 \%$ pour FPA et $76 \%$ et $77 \%$ pour FPV. Cependant, les valeurs de conductivité relevées avec FPA restent largement supérieures à celles de FPV tous les trois jours, et sachant que seules les plantes font la différences entre les deux massifs d'une part et que d'autre part $A$. gayanus a donné un système racinaire deux fois plus long et trois fois plus volumineux que celui de FPV, on peut attribuer la plus forte minéralisation du filtrat issu de FPA à un effet rhizosphère plus important lié à une meilleure colonisation du support racinaire par les bactéries. Cela est d'autant plus justifié que les racines et rhizomes offrent une plus grande surface de croissance aux bactéries et une plus grande aération de la zone qui constitue également un passage préférentiel de l'eau chargée de nutriments pour les bactéries, qui par voie de conséquence feront preuve d'une activité enzymatique plus importante (Liénard et al., 2005). Des travaux antérieurs ont montré que les éléments alcalins et alcalino-terreux que sont $\mathrm{Na}, \mathrm{Ca}$ et $\mathrm{Mg}$ sont très peu utilisés par la plante (Gu et al., 2006 ; Kohler et al., 2004), leurs teneurs sont essentiellement affectées par l'adsorption sur le support granulaire qui peut se retrouver saturé avec le temps. Xu et Jaffè (2006) ont eux aussi noté un impact très important de l'évapotranspiration sur la concentration de certains éléments par traitement des eaux usées par filtre planté à écoulement vertical. Les travaux de Morani et Giardini (2009) faisant état d'une augmentation des concentrations de $89 \%$ en $\mathrm{Na}$ et $74 \%$ en $\mathrm{Mg}$ par rapport aux valeurs d'entrées, confirment nos résultats. L'importance de l'évapotranspiration plus forte le premier jour du fait du stress hydrique des quatre jours de repos, se traduit par une diminution des débits restitués plus marquée ce jour. Cela explique la décroissance des teneurs en sels minéraux quand on passe du premier au troisième jour. A contrario, les travaux de Ouattara et al. (2008) sur panicum maximum, font état d'une baisse de la conductivité par rapport aux valeurs de l'effluent d'entrée, qu'ils attribuent à une rétention des sels par le massif essentiellement. Résultat contraire à ceux obtenus par Coulibaly 
et al. (2008) qui ont relevé une augmentation de la conductivité avec le même type de sable. Cette différence de comportement pourrait être attribuée au type de plante utilisé, mais également à la typologie des eaux d'alimentation.

\section{Conclusion}

Les résultats obtenus à l'issue de cette étude montrent que les abattements sur les paramètres de pollution organique sont satisfaisants grâce à l'action conjuguée des macrophytes, des bactéries et de la barrière physique que constitue le massif de sable. Cependant, nous pensons que travailler sur plusieurs années peut permettre de dégager le comportement dans la durée des plantes utilisées en termes de performances épuratoires et d'adaptabilité aux conditions climatiques mais également de charges organiques et hydrauliques croissantes, car des travaux antérieurs ont montré que les performances des plantes s'améliorent de la première à la deuxième année (Morari et al., 2009 ; Solano et al., 2004), ce avec le développement du système racinaire. Néanmoins pour une première approche, $V$. nigritana semble être plus résistante face au stress hydrique tandis que $A$. gayanus offre de meilleures capacités épuratoires par un prélèvement plus important de nutriments ( $\mathrm{N}$ et $\mathrm{P}$ ) et sur l'élimination de la pollution carbonée en termes de charges rejetées, tandis que lorsqu'on considère les teneurs en azote éliminé, les deux réacteurs ont des performances peu différenciées ( $p>0,05$ pour $\mathrm{NO}_{3}, \mathrm{NH}_{4}$ et $\mathrm{NK}$ ). De façon générale, les cultures fixées sur supports fins sont un meilleur moyen d'assurer de bons abattements sur la pollution organique en traitement secondaire ou tertiaire. Cependant, la forte évapotranspiration sous climat sahélien entraine une réduction non négligeable des débits avec pour conséquence une augmentation des concentrations en sels minéraux. L'expérience menée permet ainsi de dire que sous climat soudano-sahélien il n'est pas judicieux d'envisager un temps de repos double du temps d'alimentation comme cela est généralement préconisé en France, puisqu'à temps de repos à peine supérieur (4 jours), le stress hydrique a un impact important sur la résistance des plantes, les charges hydrauliques restituées et la qualité du filtrat. Aussi, une réduction plus importante du temps de repos pourrait-elle constituer une solution à même d'induire une baisse des teneurs en sels minéraux et permettre la restitution de débits hydrauliques plus importants susceptibles d'être réutilisés en agriculture, étant donné que le déficit hydrique est un problème récurrent dans les pays sahéliens.

\section{ACKNOWLEDGEMENTS}

Nous adressons nos remerciements à la Fondation Internationale pour la Science IFS (Grantee No: W/4265-1) et à l'ambassade de France au Burkina Faso pour le financement de ces travaux de recherche. Nos remerciements s'adressent également à Alain LIENARD, chercheur au CEMAGREF de Lyon pour ces conseils avisés.

\section{REFERENCES}

Agence de l'eau, Groupe Macrophytes et Traitement des Eaux. 2005. Epuration des eaux usées domestiques par filtres plantés de macrophytes: Recommandations techniques pour la conception et la réalisation. Version 1.

Bilgo A, Masse D, Sall S, Serpantié G, Chotte J-L, Hien V. 2007. Chemical and microbial properties of semiarid tropical soils of short-term fallows in Burkina Faso, West Africa. Biol. Fertil. Soils, 43: 313-320.

Champagnat P, Figueredo G, Carnat AP, Carnat A, Lamaison JL. 2006. Acides gras et insaponifiables d'extraits obtenus à partir des sommités fleuries et des rhizomes de Vetiveria nigritana (Benth.) Stapf, Poaceae. OCL.,13(2-3): 190-194.

Coulibaly L, Assamoi BA, Savane I, Bado Y. 2008. Un filtre à sable perforé à alimentation intermittent pour l'épuration des effluents d'huilerie de palme : Cas de l'unité industrielle de Toumanguié, Côte d'Ivoire. Eur. J. Sci. Res., 20(2): 226-238.

$\mathrm{Gu}$ BH, Chimney MJ, Newman J, Nungesser MK. 2006. Limnological characteristics of 
a subtropical constructed Wetland in south Floride (USA). Ecol. Eng., 27: 345-360.

Heller R, Esnault R, Lance C. 1998. Physiologie Végétale. Nutrition (Tome 1, $6^{\mathrm{e}}$ édn). DUNOD, Collection Sciences Sup.

Kantawanichkul S, Kladprasert S, Brix H. 2009. Treatment of high-strength wastewater in tropical vertical flow constructed wetlands planted with Typha angustifolia and Cyperus involucratus. Ecol. Eng., 35(2): 238-247.

Kengne IM, Akoa A, Soha EK, Tsama V, Ngoutane MM, Dodane P-H, Koné D. 2008. Effects of faecal sludge application on growth characteristics and chemical composition of Echinochloa pyramidalis (Lam.) Hitch. and Chase and Cyperus papyrus L. Ecol. Eng., 34: 233-242.

Kohler EA, Poole VI, Reicher, ZJ. 2004. Nutrient, metal and pesticides removal during storm and non storm events by constructed wetland on an urban golf course. Ecol. Eng., 23: 285-288.

Liénard A, Molle P, Boutin C, Dodane PH. 2005. Traitement des eaux usées par marais artificiels: action des plantes et développement de la technique en France. TSM., 1. $100^{\text {ème }}$ année.

Molle P, Liénard A, Boutin C, Merlin G, Iwema A. 2004. Traitement des eaux usées domestiques par marais artificiels : état de l'art et performances des filtres plantés de roseaux en France. Ingénierie, $\mathrm{N}^{\circ}$ spécial. 23-31.

Molle P. 2003. Filtres plantés de roseaux: limites hydrauliques et rétention $\mathrm{du}$ phosphore. PhD thèse, université de Montpellier II, Montpellier, P. 217.

Morani F, Giardini L. 2009. Municipal wastewater treatment with vertical flow construted wetlands for irrigation. Ecol. Eng., 35: 643-653.

Münch C, Kuschk P, Röske I. 2004. La stimulation racinaire de l'azote concerne-telle des zones limitées ou l'ensemble des marais artificiels ? Ingénierie, $\mathrm{N}^{\circ}$ spécial. $5-11$.
Obarska-Pempkowiak H, Gajewska M. 2005. Recent developments in wastewater treatment in constructed wetlands in Poland. Modern Tools and Methods of Water Treatment for Improving Living Standard. 279-293.

Ouattara J-MP, Coulibaly L, Manizan PN, Gourene G. 2008. Traitement des Eaux Résiduaires Urbaines par un Marais Artificiel à Drainage Vertical Planté Avec Panicum Maximum sous Climat Tropical. Eur. J. Sci. Res., 23(1): 25-40.

Ouédraogo-Koné S, Kaboré-Zoungrana CY, Ledin I. 2008. Intake and digestibility in sheep and chemical composition during different seasons of some West African browse species. Trop. Anim. Health Prod., 40: $155-164$.

Solano ML, Soriano P, Ciria MP. 2004. Constructed Wetlands as a sustainable solution for wastewater treatment in small villages. Biosyst. Ing., 87(1): 109-118.

Tunçsiper B, Ayaz SC, Akça L. 2006. Modelling and evaluation of nitrogen removal performance in subsurface flow and free water surface constructed wetlands. Wat. Sci. Technol., 53(12): 111120.

Vymazal J. 2004. Elimination du phosphore par faucardage de la fraction aérienne de la végétation dans les marais artificiels pour le traitement des eaux usées. Ingénierie, $\mathrm{N}^{\circ}$ spécial. 13-21.

Vymazal J, Greenway M, Tonderski K, Brix H, Mander Ü. 2006. Constructed wetlands for wastewater treatment. Ecol. Stud., 190: 69-96.

Winter K-J, Goetz D. 2003. The impact of sewage composition on the soil clogging phenomena of vertical flow constructed wetlands. Wat. Sci. Technol., 48(5): 9-14.

Xu S, Jaffè PR. 2006. Effects of plants on the removal of hexavalent chromium on wetland sediments. Environ. Qual., 35: 334-341. 